\title{
Sentinel node biopsy in combination with extended pelvic lymph node dissection for men with prostate cancer at risk of nodal involvement
}

\author{
Mauro Gacci, Pietro Spatafora, Sergio Serni \\ Department of Urology, University of Florence, Careggi Hospital, Florence, Italy \\ Correspondence to: Mauro Gacci. AOUC, Largo Brambilla 3 Urologic Clinic, San Luca Nuovo, 50134 Florence, Italy. Email: maurogacci@yahoo.it. \\ Comment on: Wit EM, Acar C, Grivas N, et al. Sentinel node procedure in prostate cancer: a systematic review to assess diagnostic accuracy. Eur Urol \\ 2016. [Epub ahead of print].
}

Submitted Nov 21, 2016. Accepted for publication Nov 29, 2016.

doi: $10.21037 /$ tcr.2016.12.63

View this article at: http://dx.doi.org/10.21037/tcr.2016.12.63

Initial diffusion of prostate cancer ( $\mathrm{PCa}$ ) occurs in the regional lymph node (LN) stations. Positive LNs represent a significant adverse prognostic factor in $\mathrm{PCa}$ and can be associated with systemic metastases $(1,2)$. Several anatomic studies with extensive lymphadenectomy have demonstrated that the obturator fossa is not always the primary site for LNs metastasis: therefore, pelvic LN dissection (LND) limited to the obturator fossa can miss about $50 \%$ of $\mathrm{LN}$ metastases. Therefore, an extended pelvic LN dissection (ePLND) should be performed in all intermediate-high-risk PCa, while a limited LND should be avoided, for the high risk of missing at least half the nodes involved (3).

An ePLND provides several prognostic data, including the number of positive LNs, the volume of metastasis within the $\mathrm{LN}$ and the penetration of the capsule of the node, which cannot be achieved with other techniques. ePLND for PCa should include not only the external and obturator regions as well as the portions medial and lateral to the internal iliac vessels, but also the common iliac LNs at least up to the ureteric crossing, thus removing approximately $75 \%$ of all nodes potentially harboring metastasis (4). The assessment of an extended lymphadenectomy is performed in relation to the template and the number of LNs removed (5). The ePLND needs of surgical skills and is time consuming.

The benefits of ePLND are accompanied by the potential intraoperative and postoperative complications. Intraoperative complications inherent to ePLND include ureteral, major vascular, and obturator nerve (sensory or motor neuropraxia) injury. Postoperative complications include symptomatic pelvic lymphocele, development of lymphoedema, ileus, deep venous thrombosis, and pulmonary embolism. The most common complication is the pelvic lymphocele. It is frequently asymptomatic and usually remains undetected. However, in some cases it is necessary to place drainage, in particular for compression phenomena on the vessels or for infections (6).

With the development of minimally invasive surgery, a simple sentinel LN biopsy (SNB) has been proposed as a novel promising technique to reduce the risk of complication, improve the staging efficacy and limit operative time. The sentinel LN is the first node that receives lymphatic drainage from the primary tumor: the concept of the SNB is based on the observation that metastases do not occur in downstream nodes if sentinel LNs are free of tumors. Sentinel guided lymphadenectomy has replaced extended lymphadenectomy in several neoplasms: in particular, for tumor such as breast cancer, penile cancer, and melanoma, SNB has become routine for nodal staging.

In 1999, Wawroschek et al. (7) started to transfer techniques and concepts of sentinel node identification to PCa. ${ }^{99 m}$ technetium nanocolloid was transrectally injected directly into the two lobe of the prostate under ultrasound guidance 1 day before pelvic lymphadenectomy. Thereafter a dynamic lymphoscintigraphy and an intraoperative identification with a gamma probe were done. Lymphatic drainage for the prostate gland is highly variable and complex, so different techniques and tracers have been tested to mark the sentinel LN in PCa. Moreover, in the last years, several methodologic improvements have been proposed, including SPECT-CT, intraoperative SN 
identification with a minimally invasive/robotic approach, the use of fluorescence imaging guided surgery, and hybrid tracers combining ${ }^{99 \mathrm{~m}} \mathrm{Tc}$ labeled colloid and indocyanine green for guidance (8).

Wit $e t$ al. has recently published a systematic review with the aim to assess the diagnostic accuracy of SNB in PCa (9). The authors have identified more than 300 articles and selected 21 studies identified from January 1, 1999 to May 31,2016 , recruiting a total of 2,509 patients. They concluded that $\mathrm{SNB}$, as a diagnostic tool, was almost equivalent to ePLND with a low non diagnostic rate (4.1), high overall sensitivity (95.2) and specificity (100.0), no false positive cases (0) and low false negative rates (4.8). Moreover, the rate of detecting positive nodes outside the ePLND template was low: therefore, SNB provided no additional diagnostic value over ePLND, although SNB appeared to increase nodal yield by increasing the number of affected nodes when combined with ePLND. The conclusion of this review was that combining SNB with ePLND could be considered as a valid option for men with high risk disease.

The strength of the review of Wit et al. is the systematic approach taken to assess and appraise the evidence base, including use of the standard methods recommended by the Cochrane Methods Group for Systematic Review of Screening and Diagnostic Tests (10), adherence to Preferred Reporting Items for Systematic Reviews (PRISMA) (11), and Standards for Reporting Diagnostic Accuracy Studies (STARD) (12) guidelines and assessment of risk of bias (RoB) using QUADAS-2. Moreover, the studies included in the review were very recent: all articles have been published within the last ten years and the majority within the last 3-5 years. The tracer used was ${ }^{99 \mathrm{~m}}$ technetium nanocolloid in almost all cases and the referral template of LND was the ePLND.

However, the review has also several limitations. Only in five studies' authors reported data from more than 100 patients, that should be considered as a minimal population to reduce the RoBs related with characteristics of both patient (age, BMI, comorbidity, prostate and lymphatic drainage characteristics) and center (urologists and pathologists). Moreover, half of the patients included in this review were not limited to intermediate/high risk or only high risk PCa according to the D'Amico risk group classification. As reported in EAU Guidelines, LND should be avoided for low risk PCa (3): the extreme rare cases of positive LNs in this very favorable group of patients may affect the results on the whole population. Finally, regarding the median number of LNs removed, 3 articles did not report the data, while for 7 the median number was below 14. As reported by Abdollah et al. (5) 14 LNs should be considered as a prognostic relevant cut-off point in order to define an extended lymphadenectomy: in particular, in patients with $\mathrm{LN}$ involvement the removal of at least 14 LNs during radical prostatectomy was associated with a significant improvement in cancer-specific survival rate (5).

Nevertheless, the interesting review from Wit et al. confirms an urgent need to standardize the definition and the technique of SNB: unifocal vs. multifocal PCa, intratumoral $v s$. peritumoral injection, monolateral $v s$. bilateral inoculation can strongly affect the definition of primary lymphatic landing sites (13). Therefore, patients with high risk of nodal involvement should undergo an extended PLND, based on a template including common iliac arteries up to the cross with ureter obturator region and LNs medial and lateral of the internal iliac vessels. As suggested by the authors, SNB in combination with ePLND seems to provide a better nodal removal by increasing the number of affected nodes in $5 \%$ of cases in high risk $\mathrm{PCa}(9)$.

Despite the recent methodologic advances in SNB techniques, a prospective well designed trial comparing $\mathrm{SN}$ dissection in addition to or alternatively to ePLND has not yet been performed. Many clinicians claim against $\mathrm{SN}$ detection in PCa mainly due to the multifocality of this specific neoplasm, the lack of a single positive sentinel node, the difficulty of peritumoral tracer inoculation and the lack of a reliable technique for intraoperative detection of the sentinel node. Future studies are strongly recommended to define if SNB with ePLND could allow improving the oncologic outcomes, in addition to the hypothetic role in staging of PCa.

\section{Acknowledgments}

Funding: None.

\section{Footnote}

Provenance and Peer Review: This article was commissioned and reviewed by the Section Editor Hong-Chao He, MD, $\mathrm{PhD}$ (Department of Urology, Shanghai Ruijin Hospital, Shanghai Jiaotong University School of Medicine, Shanghai, China).

Conflicts of Interest: All authors have completed the ICMJE uniform disclosure form (available at http://dx.doi. org/10.21037/tcr.2016.12.63). The authors have no conflicts of interest to declare. 
Ethical Statement: The authors are accountable for all aspects of the work in ensuring that questions related to the accuracy or integrity of any part of the work are appropriately investigated and resolved.

Open Access Statement: This is an Open Access article distributed in accordance with the Creative Commons Attribution-NonCommercial-NoDerivs 4.0 International License (CC BY-NC-ND 4.0), which permits the noncommercial replication and distribution of the article with the strict proviso that no changes or edits are made and the original work is properly cited (including links to both the formal publication through the relevant DOI and the license). See: https://creativecommons.org/licenses/by-nc-nd/4.0/.

\section{References}

1. Schiavina R, Gacci M, Briganti A, et al. Can side-specific biopsy findings predict the side of nodal metastasis in clinically localized prostate cancer? Results from a multicenter prospective survey. Eur J Surg Oncol 2013;39:1019-24.

2. Schiavina R, Bianchi L, Borghesi M, et al. Predicting survival in node-positive prostate cancer after open, laparoscopic or robotic radical prostatectomy: a competing risk analysis of a multi-institutional database. Int J Urol 2016;23:1000-8.

3. Mottet N, Bellmunt J, Bolla M, et al. EAU-ESTRO-SIOG guidelines on prostate cancer. Part 1: screening, diagnosis, and local treatment with curative intent. Eur Urol 2016. [Epub ahead of print].

4. Mattei A, Fuechsel FG, Bhatta Dhar N, et al. The template of the primary lymphatic landing sites of the prostate should be revisited: results of a multimodality

Cite this article as: Gacci M, Spatafora P, Serni S. Sentinel node biopsy in combination with extended pelvic lymph node dissection for men with prostate cancer at risk of nodal involvement. Transl Cancer Res 2016;5(Suppl 7):S1377-S1379. doi: $10.21037 /$ tcr.2016.12.63 mapping study. Eur Urol 2008;53:118-25.

5. Abdollah F, Gandaglia G, Suardi N, et al. More extensive pelvic lymph node dissection improves survival in patients with node-positive prostate cancer. Eur Urol 2015;67:212-9.

6. Ploussard G, Briganti A, de la Taille A, et al. Pelvic lymph node dissection during robot-assisted radical prostatectomy: efficacy, limitations, and complications-a systematic review of the literature. Eur Urol 2014;65:7-16.

7. Wawroschek F, Vogt H, Weckermann D, et al. The sentinel lymph node concept in prostate cancer - first results of gamma probe-guided sentinel lymph node identification. Eur Urol 1999;36:595-600.

8. Ramírez-Backhaus M, Mira Moreno A, Gómez Ferrer A, et al. Indocyanine Green Guided Pelvic Lymph Node Dissection: An Efficient Technique to Classify the Lymph Node Status of Patients with Prostate Cancer Who Underwent Radical Prostatectomy. J Urol 2016;196:1429-35.

9. Wit EM, Acar C, Grivas N, et al. Sentinel Node Procedure in Prostate Cancer: A Systematic Review to Assess Diagnostic Accuracy. Eur Urol 2016. [Epub ahead of print].

10. Irwig L, Glasziou P. Cochrane Methods Group on systematic review of screening and diagnostic tests: recommended methods. Cochrane Collaboration 1996.

11. Moher D, Liberati A, Tetzlaff J, et al. Preferred reporting items for systematic reviews and meta-analyses: the PRISMA statement. PLoS Med 2009;6:e1000097.

12. Bossuyt PM, Reitsma JB, Bruns DE, et al. STARD 2015: an updated list of essential items for reporting diagnostic accuracy studies. BMJ 2015;351:h5527.

13. Zehnder P, Studer UE. Editorial comment. Urology 2012;80:1087. 